\title{
David Salomon
}

\section{Ultraimperialismus oder interimperialistische Konkurrenz?}

\author{
Fragen an eine aus den Fugen geratene Zeit
}

\section{1 oder ein Imperialismusdiskurs des Mainstreams}

In den Jahren nach den Anschlägen auf das World Trade Center und das Pentagon am 11. September 2001 erlebten die Begriffe „Empire“ und „Imperialismus“ ein Comeback. In der Zeitschrift Foreign Affairs sprach der Princeton-Professor John G. Ikenberry von „America’s Imperial Ambition“ (Ikenberry 2002). Der liberale Politikwissenschaftler Michael Ignatieff diagnostizierte zustimmend ein „Empire lite“, in dem der „Menschenrechtsanwalt als Imperialist“ (Ignatieff 2003: 45) auftrete, und Max Boot, bis 2002 Redakteur des Wall Street Journal, postulierte in seinem „Plädoyer für ein Empire“: „So liegt es nicht nur im Interesse der Afghanen, sondern auch in unserem ureigensten Interesse, dass wir das Land wieder aufbauen, dass wir dort die Entstehung von Demokratie fördern und sicherstellen, dass das Land nie wieder Terrorismus exportiert." (Boot 2003: 70) Ähnlich argumentierte seinerzeit auch der Kommunitarist Michael Walzer, der einen „guten Imperialismus" ausmachte und betonte: „Wir haben zwangsläufig diese Rolle übernommen, weil andere sie uns überlassen haben. Wir sind die verantwortliche Macht, die die Gefahren in der Welt meistern muss." (Walzer 2003: 54)

Dieser Diskurs fand auch in Europa Widerhall. Im Juli 2003 zitierte die Frankfurter Allgemeine Zeitung in ihrer Rubrik „Die Stimmen der Anderen“ den französischen Figaro mit dem Satz, George W. Bush entdecke „gerade das Paradox des Imperialismus. Wie einst (der britische Autor) Rudyard Kipling sagte, als er die 'Bürde des weißen Mannes' feierte, kann es einen nachhaltigen Sieg nur geben, wenn der Eroberer zum Diener seiner Gefangenen wird. Zwei Monate nach ihrem Einzug in Bagdad finden sich die Amerikaner, die doch gefeierte Befreier sein wollten, als verhaßte Besatzer wieder. [...] Angesichts der Größe dieser Aufgabe wird sich Amerika plötzlich bewußt, daß es trotz seiner Macht nicht alles allein machen kann." (Figaro 2003: 2) Im März 2003 findet sich an gleicher Stelle ein Auszug aus einem Artikel in der dänischen Zeitung Politiken, der dem seinerzeitigen stellvertretenden US-Verteidigungsminister Paul Wolfowitz 
- „sicherlich das nachdenklichste und visionärste Mitglied der amerikanischen Regierung “ - attestierte, dass ,sein ,demokratischer Imperialismus' im Irak eine entwicklungspolitische Perspektive enthält" (Politiken 2005: 2). Verständnisvoll zeigte sich auch Thomas Schmid in der Frankfurter Allgemeinen Sonntagszeitung: „Der Irak-Krieg ist nicht defensiv, sondern offensiv, er ist ein imperialistischer Krieg. Deswegen wird er als Rückfall, als Atavismus, als Wiederkehr einer überwunden geglaubten dunklen Vergangenheit angesehen. Vielleicht aber hat die amerikanische Regierung ja gute Gründe für ihren Weg gehabt." (Schmid 2003: 13) Und Alan Posener, Kommentarchef der Welt am Sonntag, veröffentlichte ein - nach der Aufnahme in die Schriftenreihe der Bundeszentrale für politische Bildung weitverbreitetes - Buch unter einem Titel, der den US-amerikanischen Diskurs unverblümt den Europäern als Programm andiente: „Imperium der Zukunft - Warum Europa Weltmacht werden muss" (Posener 2007).

Auffallend an dieser Zusammenstellung ist, dass über Imperialismus nicht nur von den „üblichen Verdächtigen“ gesprochen wurde, sondern dass der Begriff im „Mainstream“ der Debatte um eine „Neue Weltordnung“ angekommen war. Auf beiden Seiten des Atlantiks wurde eine Argumentationsstrategie salonfähig, die das Imperiale als Selbstbeschreibung der Weltpolitik „des Westens“ zu rehabilitieren suchte und dabei auf Ideologeme zurückgriff, die bereits im kolonialen Kontext des ausgehenden 19. Jahrhunderts von Verfechtern eines „ethischen Imperialismus“ ins Feld geführt wurden. (vgl. Deppe et al. 2011: 103ff.) Machtpolitisches Eigeninteresse der führenden kapitalistischen Staaten und das universalistische Weltinteresse nach einer Zivilisierung der zwischen- und innerststaatlichen Politiken, nach effektiver Durchsetzung von Menschenrechten und säkularer Moral bilden demnach keinen Gegensatz, sondern finden in der deklarierten Verantwortung der „Starken“ gegenüber den „Schwachen“ einen gemeinsamen, wenngleich in sich widersprüchlichen Nenner. In dieser Konstellation ergänzten sich zwei interessenpolitische Strategien: Einerseits bildete er programmatische Imperialismus das Gegenprogramm zum weitgehenden Rechtsuniversalismus, der nach dem Zweiten Weltkrieg zur Gründung der Vereinten Nationen, zur grundlegenden Reform des Völkerrechts und zur Ächtung des Krieges als Mittel der Politik geführt hatte. Andererseits blieb die EmpireDebatte, die auch im politischen und sozialwissenschaftlichen Mainstreams geführt wurde, anschlussfähig an jene Diskurse, die im Ende der Blockkonfrontation und in der kapitalistischen Globalisierung die Chance zu einer weltweiten liberalen Demokratie nach westlichem Vorbild ausgemacht hatte. Gerade in seinem Oszillieren zwischen dem idealistischen und dem realistischen Paradigma internationaler Politik konnte der neue Empire-Diskurs den imperialistischen Krieg an der Peripherie salonfähig machen und zugleich den Eindruck erwecken, mit den alten Formen bloßer Interessenpolitik gebrochen zu haben. 


\section{Marxistische Imperialismustheorien als Zeitdiagnostik}

Beim Reden über „Imperialismus“ gilt es zunächst zwischen dem Gebrauch des Begriffs in der politischen Rhetorik und seiner Verwendung im Kontext wissenschaftlicher Analysen zu unterscheiden. Die klassischen Imperialismustheorien, die in einer ,ersten Welle“ - um die Wende vom 19. in das 20. Jahrhundert - entstanden, verknüpften analytisch drei Aspekte: Erstens fragten sie - am Vorabend des Ersten Weltkriegs -nach dem Zusammenhang von Kapitalismus und Kolonialismus, nach den Machtbeziehungen zwischen den kapitalistischen Kernländern, und damit nach den ökonomischen und interessenpolitischen Ursachen des Militarismus und der Aufrüstungspolitik. Zweitens verhandelten sie die grundlegende Frage nach dem Kapitalismus dieser Epoche. Spätestens mit Lenins Schrift Der Imperialismus als höchstes Stadium im Kapitalismus war der Name endgültig als Periodisierungsbegriff für eine spezifische Entwicklungsstufe kapitalistischer Produktionsweise eingeführt. Drittens schlossen die meisten frühen (und auch die späteren) Imperialismustheorien die systematische und historische Kritik am analysierten Gegenstand ein. Dies galt nicht allein für marxistische Autoren (neben Lenin 1917 sind hier insbesondere Rosa Luxemburg 1913, Karl Kautsky 1914, Michail Bucharin 1917 und Rudolf Hilferding 1910 zu nennen), sondern auch für bürgerliche Imperialismustheoretiker wie John A. Hobson (1902) und Hannah Arendt (1951). ${ }^{2}$ Auch wenn nicht alle diese Theorien auf die Überwindung des Kapitalismus als System zielten, stimmten sie doch zumeist in der Erkenntnis überein, dass eine Überwindung des Imperialismus zumindest mit einer Einschränkung kapitalistischer Verfügungsgewalt einhergehen müsse. Angesichts des Entwicklungstands der zwischenimperialistischen Konkurrenzbeziehungen, der schon vor dem Ersten Weltkrieg von der Herausbildung großer nationaler Schwerindustriekonglomerate geprägt war, kann die Ineinssetzung von Periodisierungs- und Imperialismustheorie durchaus überzeugen; der Einfluss dieser theoretischen Verknüpfung lässt sich bis in die Faschismustheorie Dimitroffs, aber auch bei der Frankfurter Schule nachzeichnen. Nach dem Ende der Periode „rivalisierender Imperialismen“ (Cox 1987: 151) und der Etablierung einer bipolaren Weltordnung traten allerdings die analytischen Schwerpunkte, die den Kern der klassischen Imperialismustheorien ausgemacht hatten, zunehmend auseinander.

Die Veränderungen der Produktionsweise blieben der Gegenstand von Analysen, die sich an Lenins Imperialismustheorie orientierten, so etwa in der Theorie

1 In einem Entwurf zum Stichwortartikel Imperialismus für das Historisch-Kritische Wörterbuch des Marxismus (HKWM) unterschied Jan Otto Andersson drei Wellen imperialismustheoretischer Debatte (Andersson 2001).

$2 \mathrm{Zu}$ diesen ersten marxistischen Debatten vgl. Deppe et al. 2004: 17ff.; Deppe et al. 2011: 27ff.; Salomon 2014. Zu Hobson und Arendt vgl. Deppe et al. 2011: 14.. 
des Staatsmonopolistischen Kapitalismus $(\mathrm{SMK})^{3}$. Denselben Ausgangspunkt wählten aber auch neuere theoretische Ansätze wie die Regulationstheorie. In dieser „zweiten Welle“ des imperialismustheoretischen Diskurses traten neue Aspekte in den Vordergrund, die sich vor allem im Begriff der Dependenz zusammenfassen. Sowohl in den Diskussionen um Imperialismus im engeren Sinn wie in den normativen, antiimperialistisch geprägten Ansätzen wurden zunehmend die Ungleichheits- und Abhängigkeitsverhältnisse zwischen kapitalistischem Zentrum und Peripherie (vgl. Senghaas 1973) thematisiert, die auch nach der Auflösung der alten Kolonialreiche bestehen blieben. Zentrale Bezugspunkte des Antiimperialismus waren vor allem die offenen (Vietnam) und verdeckten (v.a. Lateinamerika) Kriege der USA und „des Westens“ an der Peripherie, wogegen sich auf der ganzen Welt eine starke Friedensbewegung formierte. Der alte Ost-West-Gegensatz fungierte dabei als Matrix der Analyse internationaler Beziehungen - mitunter um den Preis einer Vernachlässigung der inneren gesellschaftlichen Verhältnisse in den peripheren Staaten.

Ein ungeahnt 'friedliches' Element erhöhte die Komplexität der zu analysierenden Veränderungen. Während sich der die Weltordnung prägende Zentralkonflikt als Gegensatz unterschiedlicher Wirtschafts- und Gesellschaftssysteme darstellte, kam es zwischen den (atomar hochgerüsteten) kapitalistischen Zentren zu einer tief greifenden ökonomischen Verflechtung, die einen Rückfall in alte Formen der zwischenimperialistischen Konkurrenz eher unwahrscheinlich machte. Insbesondere in den 1960er Jahren explodierten die US-amerikanischen Direktinvestitionen in Europa (Poulantzas 1973: 32f.). Auch als in den 1970er Jahren das System von Bretton Woods zusammenbrach und der fordistisch geprägte Nachkriegskapitalismus in die Krise geriet, blieben tiefergehende Verwerfungen zwischen den westlichen kapitalistischen Hauptländern aus - obschon das Überdauern der Weltmachtstellung der USA während der ersten Decline-Debatte (Kennedy 2000) in den 1980er Jahren Gegenstand heftiger Kontroversen war. Mit der schrittweisen Durchsetzung neoliberaler Strategien in der Wirtschafts- und Gesellschaftspolitik und vor allem nach dem Zusammenbruch des Ostblocks kam in jener kapitalistischen Periode, die Paul Windolf (2005) als „Finanzmarktkapitalismus“ bezeichnet, zu einer Revitalisierung der ökonomischen Vormachtstellung des US-Kapitalismus in der Welt. In den 1990er Jahren spielte der Begriff des Imperialismus auch in marxistischen Zeitdiagnosen kaum mehr eine Rolle, obwohl das Jahrzehnt von zahlreichen militärischen Auseinandersetzungen (Zweiter Golfkrieg, Balkankriege) geprägt war. Der Imperialismusbegriff verschwand zwar nicht, bliebt aber weit eher als politisch-rhetorischer denn als

3 Vgl. Boccara 1973; Maier et al. 1965; Maier et al. 1971; Schleifstein/Jung 1979; vgl. Sweezy und Baran 1966. 
wissenschaftlich-analytischer Begriff in Gebrauch. Dies war nicht zuletzt dem Umstand geschuldet, dass nach der deutschen Wiedervereinigung der von manchen befürchtete Rückfall in zwischenimperialistische Gewaltpolitik ausblieb.

Noch im Jahr 2000 erklärten Antonio Negri und Michael Hardt (2002), der „alte“ Imperialismus sei im Zeitalter der Globalisierung an ein Ende gekommen. Das „Empire“, das ihn ablöse, bedeute vielmehr das Ende der klassischen Staatensouveränität überhaupt und werde den alten Gegensatz von innen und außen überwinden. Der Empire-Band lässt sich fraglos als Initialtext der „dritten Welle“ imperialismustheoretischer Diskussion seit den Nullerjahren interpretieren. Insbesondere nach dem 11. September 2001 nahm die marxistische Imperialismusdebatte erneut Fahrt auf. Dem herrschenden Diskurs gegenüber hielten - so unterschiedlich ihre Positionen im Einzelnen auch waren - Autoren wie David Harvey (2005) oder Leo Panitch und Sam Gindin (2009) am Zusammenhang zwischen imperialistisch ausgreifender Gewaltpolitik und Kapitalismus fest. Zugleich nahmen die Protagonisten in diesem Strang der Debatte von der Vorstellung Abschied, der „Imperialismus“ eigne sich nach wie vor übergreifend als zeitdiagnostischer Periodisierungsbegriff für den Kapitalismus. So spricht David Harvey explizit von einem „neuen Imperialismus“, dessen neoliberalen Kern die - gegebenenfalls gewalttätig durchgesetzte - „Akkumulation durch Enteignung“ verkörpere. ${ }^{4}$ Während Harvey die neue Qualität der Gewaltförmigkeit seit der Regierungszeit von Bush jr. für einen Ausdruck der tendenziellen Schwäche der Vereinigten Staaten hielt, betonten Panitch und Gindin die unangefochtene Vormachtstellung der USA, die ökonomisch, politisch und militärisch gleichsam ein "Globalstaat" geworden seien. Die starke Verflechtung des transatlantischen Kapitals weise Ähnlichkeiten mit dem einst von Kautsky in die Debatte eingebrachten Bild des „Ultraimperialismus“ auf (Kautsky 1914). Im Unterschied zu Kautskys dystopischer Vision handle es sich allerdings keineswegs um ein Bündnis unter Gleichen, sondern um eine asymmetrische Machtstruktur, in der die USA die Rolle des ideellen Gesamtkapitalisten übernommen hätten. Diese US-amerikanische Debatte stand der Formulierung Pate, Imperialismus sei die offene oder latente Gewaltpolitik zur externen Absicherung eines internen Regimes (Deppe et al. 2004: 17). Mit der Konzentration auf den Aspekt der Gewaltpolitik trugen die neuen Analysen der Erkenntnis Rechnung, dass sich Imperialismus als Epochenbegriff verbraucht hatte - unabhängig, ob es sich um direkte militärische Gewalt oder um den Einsatz von Erpressungsmitteln handele, um Staaten zu zwingen, eine den westlichen Kapitalinteressen gemäße Wirtschaftspolitik zu betreiben. Zugleich

4 Harvey knüpft hier an Luxemburgs These an, wonach die von Marx „sogenannte ursprüngliche Akkumulation“ nicht ein einmaliges Vorkommnis am Anfang der Geschichte des Kapitalismus gewesen sei, sondern als enteignende Inwertsetzung permanent vorkomme. 
stellte sich diese Definition in die Traditionslinie der klassischen Debatte, ging es doch um die Absicherung eines politisch-ökonomischen Regimes - das die von Harvey als „Akkumulation durch Enteignung“ gefassten Elemente einschließt im Kontext eines nach wie vor asymmetrischen Ultraimperialismus.

Die weltpolitischen Entwicklungen insbesondere seit der Krise 2007/2008 machen es nötig, einige der damaligen Überlegungen im Lichte ihrer heutigen Erklärungskraft kritisch zu überdenken, wie Deppe es fordert: „Die geopolitischen Konflikte um eine neue Weltordnung waren in den ersten Jahren nach dem Ende des Kalten Krieges noch unscharf. Zunächst dominierte das Projekt einer 'unipolaren Weltordnung', eines 'guten US-amerikanischen Imperialismus'. Dieses Projekt radikalisierte sich nach '9/11' mit den 'Kriegen gegen den Terrorismus', die keine klaren Erfolge zeitigten und dazu die US-amerikanische Ökonomie erheblich belasteten. Im ersten Jahrzehnt des neuen Jahrtausends zeichneten sich immer deutlicher die Konturen neuer Großmachtverhältnisse in der Welt ab"(Deppe 2014: 73f.). Bereits der Aufstieg der BRIC-Staaten (Boris/Schmalz 2009) hatte abermals deutlich gemacht, dass ein Blickwinkel, der sich auf den Kapitalismus der westlichen Zentren verengt, die Eigendynamiken in anderen Weltteilen nicht erfasst. Spätestens die Weltwirtschafts- und Finanzkrise hat die These von einer nahezu unanfechtbaren ökonomischen Vormachtstellung der USA weiter erschüttert. Für die Imperialismusdebatte im engeren Sinn erweist sich nicht zuletzt das Scheitern der gewaltförmigen Weltordnungspolitik in den Nullerjahren als zentral. Schon zu ihrer Hochzeit nahmen sich die globalen Steuerungsfantasien der 'alten' Imperialisten übertrieben selbstgewiss aus - doch selbst ihre schärfsten Kritiker haben das katastrophale Ergebnis ihrer Politik, das nun ein Jahrzehnt später vorliegt, nicht vorhergesehen.

\section{Rivalisierende Imperien?}

George W. Bushs Traum von einem „Greater Middle East“ träumt heute wohl kaum jemand mehr. In Afghanistan schwelt vierzehn Jahre nach der Invasion der NATO ein Bürgerkrieg weiter, während sich die militärischen Aktivitäten der USA gegen die Taliban überwiegend nach Pakistan verlagert haben, das zum Schauplatz eines jahrelangen Drohnenkriegs geworden ist. Der Krieg gegen den Irak führte zu einer Destabilisierung des Landes und bereitete den Boden für jenen sogenannten Islamischen Staat (IS), der insbesondere im Zuge des syrischen Bürgerkriegs seinen Einfluss in der Region massiv ausbauen konnte. Nachdem in Libyen das UN-Mandat einer "Flugverbotszone“ überschritten und ein RegimeChange herbeigeführt worden ist, besteht dort kein funktionierender Staat mehr. In der Unruhezone des Mittleren Ostens konkurrieren Saudi-Arabien und der 
Iran um die regionale Vorherrschaft - ein Konflikt, in den zunehmend auch die Türkei eingreift, die gerade jene kurdischen Verbände und Organisationen bekämpft, die während des Kampfes um Kobanê die wirksamste Kraft gegen den IS waren. Von einer „imperialen Mission des Westens“ zur Verbreitung von Menschenrechten, Demokratie und „freien Märkten“ - so der übliche rhetorische Dreiklang - mag angesichts dieser Entwicklungen kaum noch jemand in den USA und Europa offen sprechen. Auch in den Diskursen um eine „stärkere Übernahme von Verantwortung" (sprich die Ausübung militärischer Gewalt) geht es eher um die Eindämmung der aus Pandoras Büchse entwichenen Dämonen. Wo im herrschenden Diskurs der Imperialismus-Begriff noch Verwendung findet, zielt er nun pejorativ auf die russische Ukraine-Politik, der vorgeworfen wird, „Machtpolitik im Stil des 19. Jahrhunderts“ zu sein - ein Vorwurf, von dem sich westliche Akteure a priori freisprechen.

Der Mittlere Osten und die Ukraine sind nicht die einzigen Konfliktzonen. Neben dem - durch die atomare Bewaffnung beider Seiten bedrohlichen - Dauerkonflikt zwischen Indien und Pakistan mehren sich Nachrichten über Spannungen zwischen China und seinen mit „dem Westen“ verbündeten Nachbarstaaten. Während sich die weltpolitische Lage im Kalten Krieg als potenziell großer, aber „eingefrorener Konflikt" charakterisieren ließ, schien nach dem Zusammenbruch des realen Sozialismus zunächst die Kooperation zwischen den kapitalistischen Zentren einerseits, zwischen alten und neuen kapitalistischen Ländern andererseits die weiterhin bestehenden Konflikte zu überlagern und einzudämmen. In der gegenwärtigen Konstellation stellt sich jedoch zunehmend die Frage, ob die Zukunft von einer Rückkehr zur zwischenimperialistischen Konkurrenz geprägt sein wird.

Anders als in früheren imperialismustheoretischen Debatten wurde in der Diskussion des vergangenen Jahrzehnts der Begriff des Imperiums - genauer: die Funktionsweise von Imperialität, also die innere Verfasstheit imperialer Gebilde - zum Gegenstand eingehender Untersuchungen (Rilling 2008: 16). Dass Imperialismus und Imperialität keine identischen Begriffe sind, darauf verweist schon die historische Tatsache, dass Imperien und Reiche lange vor dem Kapitalismus bestanden und zerfielen. Zeitweise konnte gar der Eindruck entstehen, der Zerfall der alten Imperien (etwa des Osmanischen oder des Habsburger Reiches) sei die Bedingung für die Entwicklung der moderner bürgerlichen Gesellschaft überhaupt. ${ }^{5}$ Der immer wieder gefährdete Prozess, in dem diese in Europa ihre dem Gedanken der Volkssouveränität und der Demokratie verpflichtete nationalstaatliche Gestalt annahm, stand allerdings von Anbeginn in einem Spannungsverhältnis zur staatenübergreifenden, einen Weltmarkt stiftenden Tendenz, die

$5 \mathrm{Zu}$ den oftmals widerspruchsvollen Verbindungen von Liberalismus, Bürgertum, Staat und Nation im 19. Und 20. Jahrhundert vgl. insbesondere Hobsbawm 1996. 
der kapitalistischen Produktionsweise innewohnt. Über sie schrieb Karl Marx: „Das Bedürfnis nach einem stets ausgedehnteren Absatz für ihre Produkte jagt die Bourgeoisie über die ganze Erdkugel. Überall muss sie sich einnisten, überall anbauen, überall Verbindungen herstellen." (MEW 4: 465) Diese Expansionstendenz des Kapitals ist Rainer Rilling (2008: 27) zufolge eine „Besonderheit der kapitalistischen Form von Imperialität gegenüber den vorangegangenen Imperien“, sie manifestiere sich in zwei miteinander verbundenen Merkmalen: „... erstens darin, dass sie nicht in erster Linie auf direktem Zwang beruhte, sondern auf dem indirekten Zwang des Marktes, der durch die Eigentumslosigkeit der Produzenten konstituiert und reproduziert wurde“; zweitens könne aufgrund der „institutionalisierte[n] Trennung zwischen Ökonomie und Politik [...] der Raum der ökonomischen Macht des Kapitals den Einflussraum jeder politischen und militärischen Macht bei weitem überschreiten“. Da kapitalistische Märkte ohne politische Formen der Regulation weder entstehen noch auf Dauer funktionieren können, drängt das ökonomische Kapitalverwertungsprinzips seiner inneren Logik nach dazu, im historischen Prozess jene politisch-herrschaftlichen Institutionen und 'Zentren' herauszubilden, die mit Organisation des Weltmarkts betraut sind (Arrighi 1996). Das Zeitalter „rivalisierender Imperialismen“ im Übergang vom 19. ins 20. Jahrhundert war demzufolge auch Ausdruck des Vakuums, das in der Weltökonomie nach dem Ende der britischen Steuerungsfähigkeit in der Weltökonomie entstanden war (Polanyi 1944).

„Die USA sind nach wie vor die 'Nummer 1', befinden sich aber 'im Abstieg'“, bilanziert Frank Deppe den derzeitigen Stand der Entwicklung (2014: 73). Auch potenzielle Herausforderer des US-amerikanischen Hegemonieanspruchs sind auszumachen: etwa die Europäische Union, „die vor allem durch die Osterweiterung und die Einführung des Euro zu einer potenziellen Großmacht aufgestiegen, allerdings infolge der Eurokrise seit 2010 geschwächt“ erscheint, und China, das „zu einer wirtschaftlichen und politischen Großmacht“ aufgestiegen ist, die - wie die rege chinesische Investitionstätigkeit in Afrika zeigt -das von Rilling konstatierte Spiel kapitalistischer Imperien virtuos beherrscht, durch ökonomische Ausdehnung ihr Einflussgebiet zu erweitern. Zudem belege „das Bündnis der BRICS-Staaten“, in dem sich mit China, Russland und Indien drei große „Imperien“ mit unterschiedlicher Reichweite ihres Einflusses befinden, „wie sich in den letzten Jahren zahlreiche 'Schwellenländer' aus der strukturellen Abhängigkeit (sowohl im Handel als auch bei der Verschuldung gegenüber den internationalen Banken und Finanzinstitutionen) befreit und dabei untereinander neue Formen der Wirtschaftsbeziehungen entwickelt haben“ (). Unverkennbar „zeichnen sich mithin Konturen einer multipolaren Weltordnung ab, in der es neue Großmächte, aber auch neue Allianzen geben wird“ (Deppe 2014: 73ff.). Offene Gewaltanwendung beschränkt sich derzeit, wie Deppe weiter ausführt, 
auf die Unruhezonen in jenen Regionen, in denen sich rivalisierende Interessen überschneiden: „Die aktuellen Konflikte und Kriege (in Syrien und im Irak, nunmehr in der Ukraine und im Krieg der israelischen Regierung gegen die Hamas in Gaza) sind bereits durch diese Entwicklungen überdeterminiert: durch den Übergang von einer 'chaotischen' bzw. 'turbulenten' Welt-un-ordnung zu einer Ordnung rivalisierender Imperien" (ebd.: 75). In der Europäischen Union (Griechenland) verweisen zudem erpresserische Formen der Disziplinierung einzelner kleinerer Länder darauf, in welchem Maße nun auch im angestammten Einflussgebiet die imperiale Logik von Zentrum und Peripherie etablierte demokratische Standards außer Kraft setzt.

Die derzeitige Entwicklung deutet somit - wenn auch in einem völlig veränderten politisch-ökonomischen Kontext ${ }^{6}$ - auf eine historische Konstellation hin, als nach dem Ende der britischen Hegemonie ein globales Machtvakuum entstand. Angesichts der demonstrativen Ignoranz der damaligen Bush-Regierung gegenüber völkerrechtlich geltenden Standards erklärte Giovanni Arrighi bereits im Jahr 2003: „Die USA erklären, dass alle existenten Regelungen der internationalen Beziehungen keine Gültigkeit mehr haben, und sie versuchen, neue Regelungen auf der Grundlage ihrer militärische Macht zu schaffen. Das ist ein Zeichen, dass Chaos und nicht Hegemonie einsetzt. Chaos ist nichts anderes als der Zusammenbruch von Regelwerken, die einer existierenden internationalen Ordnung zugrunde liegen." (Arrighi 2003: 97) Das Scheitern der Bush'schen „Visionen“ ist nunmehr ein deutliches Indiz für den beschleunigten Niedergang der US-amerikanischen Hegemonie - mit der Folge einer neuen Welle zwischenimperialistischer, genauer: interimperialer Konkurrenz. ${ }^{7}$ Im Rückblick hat der Zusammenbruch des realexistierenden Sozialismus nicht, wie „westliche Akteuren“" zunächst erhofft hatten, die ultraimperialistische Bindungskraft „des Westens" (unter Führung der USA) gestärkt, sondern die Erosion dieser in der Systemkonkurrenz siegreichen Gestaltungsmacht eher beschleunigt. Sowohl im transatlantischen Verhältnis als auch im Verhältnis der USA zu den wich-

6 „Im Verhältnis zur Epoche des klassischen Imperialismus hat sich freilich das Verhältnis von kapitalistischer Globalisierung und nationalstaatlicher Macht verändert. Daraus resultieren wiederum Strategien, die mehr auf die ökonomische Durchdringung von Räumen (z.B. der chinesischen oder russischen Ökonomie und Finanzwirtschaft) orientiert sind und die zugleich durch die Abhängigkeit von globalen Marktbewegungen (vor allem auf den Finanzmärkten) bestimmt werden." (Deppe 2014, S. 78)

7 Arrighi zeichnete 2003 ein eher düsteres Zukunftsszenario: „Also ist alles, was wir im Augenblick vorausahnen können, eine lange Epoche von Kämpfen zwischen der Tendenz der globalen Ökonomie, sich mit Ostasien ein neues Zentrum zu schaffen, und den USamerikanischen Ansprüchen auf den Aufbau eines Weltreiches, die darauf abzielen, eben diese Tendenz zu stoppen." (Arrighi 2003, S. 97) 
tigsten asiatischen Staaten mit kapitalistischer Wirtschaftsordnung (Japan und Südkorea) bleibt zwar das asymmetrische ultraimperialistische Bündnis noch immer intakt. Zugleich lässt sich jedoch der Erosionsprozess US-amerikanischer Hegemonie innerhalb der NATO am Oszillieren „des Westen “ "zwischen unterschiedlichen und keineswegs kohärenten strategischen Optionen im Umgang mit den Unruheregionen und den dort aufbrechenden Konflikten und Bürgerkriegen ablesen. Die politische Rhetorik spitzt sich dabei zum Teil in einer Weise zu, die weniger an den Kalten Krieg als an die vergangenen Phasen der zwischenimperialistischen Propaganda erinnern. Auch wenn mittelfristig eine direkte militärische Auseinandersetzung zwischen den multipolaren Machtblöcken unwahrscheinlich bleibt, ist die verbale Aufrüstung, vor allem in den Beziehungen „des Westens“ gegenüber Russland, kaum zu übersehen. Die Debatte darüber, wie eine antiimperialistische linke Solidaritätspolitik auf diese Tendenzen reagieren kann, hat gerade erst begonnen. So überlagert - um zwei von Gramsci und Romain Rolland stammende Aphorismus zu variieren - derzeit der „Pessimismus des Verstands“ den „Optimismus des Willens“.

\section{Literatur}

Andersson, Jan Otto (2001): Imperialismus - Text written for the Historisch-Kritisches Wörterbuch des Marxismus (second version). URL: http://www.marx-seura.kaapeli.fi/archive/ imperialism.htm, Zugriff am 23.9.2015.

Arendt, Hannah (1951): Elemente und Ursprünge totaler Herrschaft. Antisemitismus, Imperialismus, totale Herrschaft, München 2001.

Arrighi, Giovanni (1996): The long Twentieh Century. Money, power, and the origins of our times, London-New York.

- (2003): Niedergang der USA und Neue Weltordnung. Gespräch mit Amy Holmes und David Salomon. In: Z. Zeitschrift Marxistische Erneuerung 14(55): 92-98.

Boccara, Paul (1973): Der staatsmonopolistische Kapitalismus. Frankfurt/M.

Boot, Max (2003): Plädoyer für ein Empire. In: Speck, Ulrich/Sznaider, Natan (Hg.): Empire Amerika - Perspektiven einer neuen Weltordnung, München: 60-70.

Boris, Dieter/Schmalz, Stefan (2009): Eine Krise des Übergangs: Machtverschiebungen in der Weltwirtschaft. In: PROKLA. Zeitschrift für kritische Sozialwissenschaft 39(4): 625-643.

Bucharin, Nikolai (1917): Imperialismus und Weltwirtschaft, Frankfurt/M 1969.

Cox, Robert W. (1987): Production, power, and world order. Social Forces in the Making of History, New York.

Deppe, Frank (2014): imperialer realismus? über eliten, experten und journalisten und die „neue deutsche verantwortung", Hamburg.

-/Heidbrink, Stephan/Salomon, David/Schmalz, Stefan/Schoppengerd, Stefan/Solty, Ingar (2004): Der neue Imperialismus, Heilbronn.

-/Salomon, David/Solty, Ingar (2011): Imperialismus, Köln.

Figaro (2003): Schwieriger Imperialismus. In: Frankfurter Allgemeine Zeitung, 3.7.2003: 2.

Harvey, David (2005): Der neue Imperialismus, Hamburg.

Hilferding, Rudolf (1955): Das Finanzkapital. Eine Studie über die jüngste Entwicklung des Kapitalismus, Berlin. 
Hobsbawm, Eric J. (1996): Nationen und Nationalismus. Mythos und Realitiät seit 1780, München. Hobson, John Atkinson (1902): Der Imperialismus, Köln-Berlin 1968.

Ignatieff, Michael (2003): Empire lite - Die amerikanische Mission und die Grenzen der Macht, Hamburg.

Ikenberry, G. John (2002): America’s Imperial Ambition. In: Foreign Affairs 81(5): 44-60.

Kautsky, Karl (1914): Der Imperialismus/Zwei Schriften zum Umlernen/Der imperialistische Krieg (Auszüge). In: Bollinger, Stefan (Hg.) (2004): Imperialismustheorien. Historische Grundlagen für eine aktuelle Kritik, Wien: 107-128.

Kennedy, Paul M. (2000): Aufstieg und Fall der großen Mächte. Ökonomischer Wandel und militärischer Konflikt von 1500 bis 2000, Frankfurt/M.

Lenin, Wladimir Iljitsch (1917): Der Imperialismus als höchstes Stadium des Kapitalismus. Gemeinverständlicher Abriß. In: Lenin Werke, Bd. 22, Berlin: 1960ff.: 189-309.

Luxemburg, Rosa (1913.): Die Akkumulation des Kapitals oder Was die Epigonen aus der Marxschen Theorie gemacht haben. Eine Antikritik. In: Rosa Luxemburg Gesammelte Werke, Bd. 5. Berlin 1970ff.: 413-523.

Maier, Lutz/Petrak, Heinz/Reinhold, Otto/Schwank, Karl-Heinz/Hemberger, Horst (1965): Imperialismus heute, Berlin.

-/Pfaff, Werner/Schwank, Karl-Heinz/Hahn, Wolfgang/Schmidt, Max/Petrak, Heinz et al. (1971): Der Imperialismus der BRD. Frankfurt/Main: Verlag Marxistische Blätter.

MEW, Marx, Karl/Engels, Friedrich (1848): Manifest der Kommunistischen Partei. In: Karl Marx und Friedrich Engels: Werke, Bd. 4. Berlin 1956ff.: 459-493.

Negri, Antonio/Hardt, Michael (2002): Empire - Dieneue Weltordnung, Frankfurt/M-New York.

Panitch, Leo/Gindin, Sam (2009): Globaler Kapitalismus und amerikanisches Imperium, Hamburg.

Polanyi, Karl (1944): The Great Transformation. Politische und ökonomische Ursprünge von Gesellschaften und Wirtschaftssystemen, Frankfurt/M 1997.

Politiken (2005): Demokratischer Imperialist. In: Frankfurter Allgemeine Zeitung, 19.3.2005: 2.

Posener, Alan (2007): Imperium der Zukunft. Warum Europa Weltmacht werden muss, Bonn.

Poulantzas, Nicos (1973): Die Internationalisierung der kapitalistischen Verhältnisse und der Nationalstaat. In: Hirsch, Joachim/Jessop, Bob/Poulantzas, Nicos: Die Zukunft des Staates, Hamburg 2001: 19-69.

Rilling, Rainer (2008): Risse im Empire, Berlin.

Salomon, David (2014): Kapitalismus und Aggression - Anmerkungen zu klassischen Imperialismustheorien. In: Steffek, Jens/Holthaus, Leonie (Hg.): Jenseits der Anarchie. Weltordnungsentwürfe im frühen 20. Jahrhundert, Frankfurt/M: 124-151.

Schleifstein, Josef/Heinz, Jung (1979): Die Theorie des staatsmonopolistischen Kapitalismus und ibre Kritiker, Frankfurt/M.

Schmid, Thomas (2003): Gewalt kann Frieden stiften. In: Frankfurter Allgemeine Sonntagszeitung, 30.3.2003: 13.

Senghaas, Dieter (1973): Imperialismus und strukturelle Gewalt. Analysen über abhängige Reproduktion, Frankfurt/M.

Sweezy, Paul M./Baran, Paul A. (1966): Monopolkapital. Ein Essay über die amerikanische Wirtschafts- und Gesellschaftsordnung, Frankfurt/M 1973.

Walzer, Michael (2003): „Die Europäische Außenpolitik kann sich nicht sehen lassen“. Interview. In: Böhnel, Max/Lehmann, Volker (Hg.): American Empire, no thank you. Andere Stimmen aus Amerika, Berlin: 47-55.

Windolf, Paul (2005): Was ist Finanzmarkt-Kapitalismus? In: Ders. (Hg.): Finanzmarkt-Kapitalismus. Analysen zum Wandel von Produktionsregimen, Wiesbaden: 20-57. 